\title{
CREENCIAS SOBRE LAS PRUEBAS UTILIZADAS PARA EVALUAR EL APRENDIZAJE DEL INGLÉS
}

\author{
Noé Vicente Araujo \\ Universidad de Nariño \\ viar22@hotmail.com
}

\begin{abstract}
Resumen
Este artículo se deriva de mi tesis doctoral titulada: "Las creencias sobre la evaluación del aprendizaje del inglés como lengua extranjera: hacia una evaluación proactiva". Bajo la dirección del Doctor Jesús Alirio Bastidas, el estudio se realizó en el Departamento de Lingüística e Idiomas de la Universidad de Nariño y utilizó el método fenomenológico-hermenéutico; básicamente, se llevaron a cabo entrevistas en profundidad y se complementó la búsqueda con la aplicación de algunas encuestas y el análisis de diez pruebas de inglés a las cuales aplicaron los estudiantes. Los resultados constituyen una aproximación importante hacia las ideas ocultas detrás de los exámenes analizados dando lugar a una variedad interpretativa casi inagotable; la investigación sugiere la existencia de sentidos y creencias eclécticas entre profesores y estudiantes sobre las pruebas utilizadas para evaluar el aprendizaje del inglés como lengua extranjera.
\end{abstract}

Palabras clave: pruebas, subjetividad, aprendizaje, inglés, creencias. 


\title{
BELIEFS ABOUT THE TESTS USED TO EVALUATE ENGLISH LEARNING
}

\begin{abstract}
This article is derived from my doctoral thesis entitled "Beliefs about the evaluation of learning English as a foreign language: towards a proactive evaluation." The study was conducted in the Department of Linguistics and Languages of the Universidad de Nariño, under the direction of Dr. Jesús Alirio Bastidas, using the phenomenological-hermeneutical method. In-depth interviews were conducted, and the search was complemented by the application of surveys and the analysis of ten English tests performed by students. The results constitute an important approximation to the hidden ideas behind the considered exams, giving rise to an almost inexhaustible interpretative material. The research suggests the existence of eclectic meanings and beliefs between teachers and students about the tests used to evaluate the learning of English as a foreign language.
\end{abstract}

Keywords: tests, subjectivity, learning, English, beliefs.

\section{CRENÇAS SOBRE EVIDÊNCIAS UTILIZADAS PARA AVALIAR O APRENDIZAGEM DO INGLÊS}

\section{Resumo}

Este artigo é derivado de minha tese de doutorado intitulada: “Crenças sobre a avaliação da aprendizagem do inglês como língua estrangeira: para uma avaliação proativa". Sob a direção do Dr. Jesus Alirio Bastidas, o estudo foi realizado no Departamento de Lingüística e Linguística da Universidade de Nariño e utilizou o método fenomenológico-hermenêutico; basicamente, entrevistas em profundidade foram realizadas e a busca foi complementada pela aplicação de algumas enquetes e pela análise de dez provas de inglês aplicadas pelos alunos. Os resultados constituem uma importante aproximação às ideias ocultas por trás das análises analisadas, dando origem a uma variedade interpretativa quase inesgotável; A pesquisa sugere a existência de sentidos e crenças ecléticas entre professores e alunos sobre os testes utilizados para avaliar a aprendizagem do inglês como língua estrangeira.

Palavras-chave: testes, subjetividade, aprendizagem, inglês, crenças. 


\section{Introducción}

El ser humano ha estado evaluando y evaluándose desde sus propios orígenes; aunque no de manera formal, siempre ha emitido juicios de valor sobre todo aquello que lo rodea, llámense personas, animales, plantas, alimentos, actitudes, personalidades, etc. Desde los tiempos de los emperadores chinos hasta comienzos del siglo XXI, la evaluación ha jugado un papel preponderante en la selección de seres humanos bien sea para ocupar cargos públicos o para acceder a niveles de sistemas educativos; con el transcurrir del tiempo se han ido configurando, de manera más sistemática, sistemas de evaluación que han sido introducidos en la escuela en procura de alcanzar metas y de comprobar los conocimientos aprendidos por un ser humano, después de ser sometido a un proceso de formación escolar.

Las pruebas orales o escritas, también conocidas como exámenes, se han convertido en los instrumentos de evaluación más utilizados en los distintos escenarios educativos en casi todo el mundo; en ellas, es posible estudiar su naturaleza, sus características o sus implicaciones sobre los protagonistas de los procesos de enseñanza y aprendizaje; no obstante, también se puede analizar aspectos invisibles de estas pruebas tales como los sentidos y las creencias que profesores y estudiantes tienen sobre ellas; ese fue, precisamente, uno de los propósitos de esta investigación. En el campo de la enseñanza del inglés, las pruebas también se utilizan para "medir" el aprendizaje logrado por el estudiante, pero más allá de los resultados de los exámenes propiamente dichos, se insinúa una serie de creencias, algunas de las cuales se revelan a continuación. En esta investigación se llevaron a cabo y analizaron 20 entrevistas, 33 encuestas y 10 exámenes.

\section{Metodología}

Como se indicó anteriormente, la investigación se hizo con base en un estudio fenomenológico-hermenéutico; siguiendo a Morin ${ }^{\mathbf{1}}$ analizar los datos es "extraer la manera como la persona ve su relación con el mundo, la interpretación que da a su experiencia global de vida, es decir, que subyace a su cotidianidad"; Ricard, Bo y Climent $^{2}$ proponen unos pasos que sirvieron como base en la realización de este estudio, a saber: a) transcripción de la información de forma cerrada (tomar nota de todo) tan pronto como fue posible, incluyendo incidentes en el proceso tales como errores, vacilaciones y manifestaciones no verbales (Deslauriers, 2004); b) elaboración de unidades de significado general, las cuales surgieron de las lecturas y relecturas intencionales del discurso; consiste, básicamente, en agrupar las intervenciones de tal forma que conformen una unidad de significado

1. Louis Morin, La methodologie de l'histoire de vie (Québec: Institute supérieur des sciences humaines, Université Laval Coll "instruments de travail No. 13", 1974), 19.

2. M. Ricard, R. M. Bo, y, C.I. Climent, Propuesta de Análisis Fenomenológico de los Datos Obtenidos en la Entrevista, UT. Revista de Ciències de l'Educació, Juny 113-133. 2010, http://pedagogia.fcep.urv.cat/ revistaut (29 de abril de 2014 ). 
incluyendo las ideas relacionados directamente con la investigación y aquellas que aparentemente no tienen relación alguna; c) elaboración de unidades de significado relevante. Se elaboran a partir de las unidades de significado general y su objetivo consiste en desglosar la información en busca de la esencia y el sentido del fenómeno estudiado; corresponde a la información que es pertinente para la búsqueda; d) elaboración de unidades de significado convergente. Teniendo en cuenta las unidades de significado relevante se agruparon las unidades de significado en las "categorías" previamente establecidas con base en los objetivos del trabajo; e) reflexión fenomenológica hermenéutica. Consistió en penetrar en la esencia de lo expresado por el entrevistado tratando de develar la experiencia misma de la persona, su mundo vivido en relación con el fenómeno objeto de investigación; f) conclusiones. Corresponden a las ideas que se recogen y condensan todo el proceso fenomenológico.

Como se puede apreciar, el análisis de información hermenéutico consiste, básicamente, en un proceso de filtración de información a través de "reducciones"; este proceso se complementó con el estudio pormenorizado de las encuestas y del análisis de los exámenes de muestra. Una etapa posterior a las señaladas anteriormente, fue la triangulación y confrontación de información proveniente de las diferentes fuentes.

\section{Resultados}

El análisis de la información condujo al establecimiento de categorías de análisis, una de ellas se denominó "Sentidos de las prácticas"; esta categoría hace referencia a la parte invisible del examen y a las creencias que tienen los participantes en relación con las prácticas de evaluación del aprendizaje del inglés; su estudio fenomenológico-hermenéutico permitió acceder a las ideas ocultas detrás de las pruebas y propiamente corresponde a la connotación entendida como la variedad en términos de compresión de lo expresado por el emisor. El sentido permitió dilucidar la parte que no se observa ni se escucha inicialmente, dando lugar a una variedad interpretativa profunda; también permitió al usuario del discurso crear su particular forma de apropiación del texto penetrando en aquellas brechas existentes entre el texto y el usuario: de esta manera, los sentidos de un texto o discurso son infinitos puesto que habrá tantos sentidos cuantas personas intenten comprender una emisión lingüística; por ejemplo: cuando un profesor expresa en clase: "Bueno, si no atienden, les va a ir mal", el sentido va más allá de un simple enunciado bien sea afirmativo o negativo; en este caso, constituye una amenaza que evidencia el poder que tiene el profesor en el aula y muestra su influencia en el comportamiento y en las calificaciones de los estudiantes. De esta categoría se derivaron cuatro subcategorías, cuyo estudio y resultados se detallan a continuación:

\section{JUSTICIA Y EQUIDAD EN LAS PRUEBAS}

Esta subcategoría hace referencia a las condiciones bajo las cuales los profesores utilizan las pruebas y evalúan a los estudiantes; el estudio señala que los docentes 
de inglés admiten la existencia de la subjetividad en las prácticas evaluativas, hecho que conduce a reconocer que en el ejercicio de la enseñanza, aprendizaje y evaluación manifestamos nuestra propia forma de vernos, de ver el mundo y de relacionarnos con él ${ }^{3}$; son los sentidos del texto o discurso y su complejidad los que hacen que inevitablemente estemos rodeados de subjetividades y que, por lo tanto, haya necesidad de acudir a las intersubjetividades como mecanismo de acercamiento entre posiciones aparentemente irreconciliables; no basta con reconocer la existencia de la subjetividad en las pruebas de inglés, es preciso reorientar los procesos y tomar decisiones que apunten a aminorar su efecto en los juicios valorativos. Algunos profesores admiten que hay preferencia por ciertos estudiantes, lo cual significa que no siempre utilizan los mismos criterios de justicia y equidad para evaluar; si bien es cierto las prácticas evaluativas están permeadas por varios elementos subjetivos (quien las elabora, cómo las elabora, por qué las elabora, en qué contextos, etc.), su influencia desmedida y descontrolada contribuye a seleccionar de manera invisible a los aprendices a tal punto de considerarlos como "aptos" y "no aptos"; de hecho, es una forma de someterlos al sistema educativo imbuido de poder y autoridad que forma alumnos dóciles, obedientes, expuestos permanente y eternamente a la vigilancia y al control4.

En relación con esta subcategoría, los estudiantes también manifiestan que los docentes tienen muchos prejuicios a la hora de evaluarlos y que es evidente la influencia de la subjetividad en la asignación de las calificaciones; incluso señalan que algunos docentes "miran caras" (muestran preferencias) para asignar notas. Un estudiante sostiene lo siguiente:

"hay un grado de subjetividad alto y es un inconformismo que hay en el estudiantado en general ¿no? Eh que la gente uno dice: "no ese profesor a tal persona la evalúa de una forma diferente que a mí" o "ese tal profesor mira caras porque yo tenía las mismas respuestas que mi compañero y a mi compañero le puso mejor o que tales". 0 "porque ese profesor entonces, ahh... esa compañera habla más con el profesor entonces a él si le puso buena nota y a mí no"; y yo creo que al momento de evaluar también los profesores llegan hacen esa subjetividad y dicen "bueno, yo veo a este estudiante que de pronto pone atención o que tales en clase le voy a echar una ayudita" o "a este muchacho, que casi no viene a clases, no no le voy a ayudar" Esa subjetividad a simple vista pues parece "descoherente" o irracional pero es la subjetividad"5.

Esta opinión advierte que la evaluación justa y equitativa tiene que ver con la ética del profesor, con la forma de mirar al otro sin hacer uso del poder; todos los alumnos esperan ser tratados como personas únicas y en igualdad de condiciones;

3. C. Coll, Martín, E., T. Mauri, M. Miras, J. Onrubia, I. Solé, y A. Zabala, El constructivismo en el aula. (Barcelona: Editorial Graó, Des Serveis Pedagógics Francesc Tárrega, 2007).

4 A. T. Sánchez, Aproximación a un Estudio Genealógico de la Evaluación Educativa en Colombia, segunda mitad del siglo XX. Tesis doctoral (Centro de estudios avanzados en niñez y juventud Cinde Universidad de Manizales, 2008).

5. Entrevista con estudiantes número 1, párrafo 25. Pasto, marzo de 2015. 
en efecto, la ética implica un compromiso consigo mismo y con los demás, refleja mi forma de actuar ante ellos, en otras palabras, es tratar al otro como si fuera yo mismo.

La mayoría de los docentes declaran que los alumnos son evaluados en las mismas condiciones en términos de contenidos, tiempo ylugar, ya que es un derecho y un principio ético; también señalan que hay más objetividad en las evaluaciones escritas de inglés que en las orales y que califican de manera anónima esta clase de evaluaciones. Según estas declaraciones, los estudiantes son evaluados de forma justa y equitativa teniendo en cuenta sus derechos y la ética del docente; sin embargo, otros docentes advierten que no siempre se aplican estos conceptos de justicia y equidad y que infortunadamente no siempre el aprendiz es evaluado con ecuanimidad; señalan algunos profesores la necesidad de ser flexibles en los criterios atendiendo a las necesidades de los estudiantes; éste es un punto a favor si se mira desde la complejidad del ser humano, en el sentido de reconocer las diferencias individuales y la existencia de las identidades diferentes incluso en una misma persona6; nuestro comportamiento en la universidad, en la casa o en un club de amigos, no siempre es el mismo.

Por su parte, pocos alumnos manifiestan que los profesores los evalúan con justicia y equidad y ética; la mayoría expresa que los profesores califican según la persona, cometen injusticias, prefieren a los alumnos avanzados (cuando en realidad son los que tienen dificultades quienes necesitan más oportunidades) y, en general, señalan que hay desigualdad. A partir de la experiencia de los alumnos, se infiere que los procesos evaluativos adolecen de ciertas irregularidades que los afectan (contrario a lo que expresan los profesores); otros estudiantes señalan que sólo son justas las calificaciones cuyas pruebas son de respuesta cerrada de $\mathrm{F}$ $\mathrm{y} \mathrm{V}$, a diferencia de lo que ocurre con aquellas de preguntas abiertas, en las que no hay equidad, es decir, se ven afectadas por la parcialidad del profesor.

También se insinúa en las opiniones de los estudiantes que factores como la relación del profesor con el alumno (empatía), el comportamiento del aprendiz, el género (especialmente los docentes veteranos que evalúan mejor a las mujeres) y la trampa, indefectiblemente tiene repercusión en los resultados de las evaluaciones en inglés; admiten que estos acontecimientos ocurren, en parte, porque los dicentes no se quejan y aceptan con resignación estas irregularidades; expresiones de los estudiantes como "me vio la cara" y "no nos tratan a todos por igual" en la evaluaciones, advierten sobre el estado y condición de indefensión de los alumnos ante la actitud de algunos profesores que generan, de esta manera, violencia al interior de las aulas.

\section{DIFICULTADES EN LAS PRUEBAS}

Esta subcategoría abarca algunos aspectos que inciden en los resultados que se obtienen en las pruebas, tales como el nerviosismo, la inasistencia, temor al

6. Edgar Morin, Introducción al Pensamiento Complejo (Barcelona: Editorial Gedisa, 2011). 
error, entre otros. Para algunos profesores entrevistados, las pruebas pueden ser confusas, ambiguas y subjetivas, así lo evidencia el siguiente testimonio:

[...] "a la hora de ver los resultados, yo siempre me he dado duro con ver resultados muy bajos que uno no espera. Entonces eh la evaluación a veces me ha dado... es decir eh me preocupa a veces los resultados sobre todo los resultados de las evaluaciones con respecto a lo que uno espera. Eh a la hora de planear evaluaciones sí es muy común que a veces uno no, no revisa muy bien a profundidad que es lo les está pidiendo a los estudiantes, no ve bien las instrucciones y a veces se vuelven confusos eso ha sido una cosa que ha aprendido" $[\ldots]^{7}$.

Esta observación constituye una autocrítica del docente en relación con la forma cómo elabora las pruebas de inglés; en efecto, toda práctica evaluativa es imprecisa, incompleta, indirecta, subjetiva y relativa ${ }^{\mathbf{8}}$; reconocer que las prácticas evaluativas no escapan a tales características, es un aspecto positivo en algunos de los profesores entrevistados; la subjetividad está presente en todo acto humano, especialmente en la escuela particularmente en el campo de las ciencias humanas y en la evaluación de un idioma extranjero; por otra parte, la objetividad es bastante esquiva en este sentido, sobre todo en el campo del conocimiento procedimental (saber hacer) y actitudinal (saber ser), dado el carácter único e irrepetible de cada persona y de sus manifestaciones lingüísticas; quizás se pueda hablar de objetividad relativa cuando se evalúa el conocimiento declarativo (saber qué) haciendo referencia a los datos, fechas o información muy específica.

Otra dificultad que destacan los docentes tiene que ver con la inasistencia de los estudiantes a clases; algunos consideran que quien no asiste no merece la máxima calificación (cinco) y que la asistencia a clases garantiza la aprobación de la asignatura, porque todo lo que se evalúa se hace en clase; en cierta manera, los profesores tienden a asociar asistencia con éxito o con aprendizaje, quizás porque nuestra cultura así lo ha establecido históricamente y porque la asistencia a clases es, por lo general, "obligatoria"; no obstante, estar en clase no necesariamente garantiza aprendizaje puesto que hay estudiantes que aprenden a pesar del profesor (como lo indicaron dos entrevistados) bien sea estudiando en casa o mediante el uso de las nuevas tecnologías; la inasistencia a clases no equivale a no aprender, todo obedece a las circunstancias y a las particularidades de cada caso, pues no hay estructuras y procedimientos fijos y únicos ${ }^{9}$ de aprendizaje y de evaluación de una lengua extranjera.

Algunos profesores señalan que la memoria a corto plazo es un aspecto importante en las pruebas de inglés; al respecto, habría que reflexionar sobre la manera cómo los profesores enseñan ya que es posible que, a través de sus cátedras, estén desarrollando la memoria a corto plazo y por eso los estudiantes

7. Entrevista con profesores número 2, párrafo 4. Pasto, marzo de 2015.

8. L.F. Bachman, Fundamental Considerations in Language Testing (Oxford: Oxford University Press, 1990).

9. G. Deleuze y F. Guattari, Rizoma (Medellín: Editorial Oveja Negra, 1976). 
se preparan solamente para el examen y después olvidan lo que supuestamente han aprendido; también manifiestan algunos docentes que no hay reflexión sobre los resultados de las pruebas, es decir, simplemente se hacen para certificar, para asignar un número o para acreditar el conocimiento del alumno sin que exista una reflexión sobre lo que ocurrió en el proceso previo a la evaluación; la supuesta verdad que expresa un examen no puede ser reducida a una definición unilateral de cada maestro ${ }^{10}$; es preciso entonces, reflexionar colectivamente para descubrir irregularidades y a la vez asumir responsabilidades. El tipo de evaluación irreflexiva se asocia con la medición o la calificación y con el paradigma cuantitativo propiamente dicho, en el cual predomina la cifra y el acto mecánico que no requiere mucho conocimiento o experiencia para calificarla, de hecho, su interés se orienta hacia la explicación en términos de causa-efecto ${ }^{11}$; las creencias sobre la confiabilidad y la validez de las pruebas han sido tan profundas, que muchas veces de todo se puede dudar (del alumno, de la aplicación de la prueba, de la manipulación humana, del docente), pero casi nunca del examen ${ }^{12}$.

Los alumnos entrevistados, por su parte, sostienen que el nerviosismo y el temor al error son dificultades que afectan considerablemente su desempeño en las pruebas de inglés; al respecto, cabe recordar que el pensamiento científico se construye con base en el error y que éste tiene una función positiva en el saber ${ }^{13}$ porque evita volver a cometer los mismos desaciertos y crea espacios para la innovación y reflexión permanentes. De igual manera, Morin reconoce que es un error buscar la verdad sin buscar el error ${ }^{\mathbf{1 4}}$, luego, es preciso aceptarlo como parte indispensable e inherente al proceso de enseñar, aprender y evaluar un idioma; el error proviene del profesor, del estudiante, del proceso y de la particularidad de cada instante, por eso hay que sacar provecho de su existencia.

Por otra parte, los docentes señalan que los resultados de las pruebas revelan las fallas pero también las fortalezas; según la función social de la evaluación, los exámenes se hacen sólo para detectar los defectos académicos de los alumnos y no sus potencialidades; "somos unos cazadores de errores" señala uno de los entrevistados, para indicar que casi siempre están buscando la equivocación del estudiante, no para corregirlo o hacer actividades de retroalimentación consistentes, sino para insinuar que sólo los "buenos alumnos" pueden acceder al conocimiento. Un profesor entrevistado sostiene:

"Yo creo que es la perspectiva del profesor ¿no? Cada quien, yo creo que eso es como más, más personal como lo ve cada profesor. Hay profesores que están

10. A. M. Pérez y Z. G. Bustamante, Evaluación escolar resultados o procesos. Investigación, reflexión y análisis crítico (Bogotá: Cooperativa Editorial del Magisterio, 2002).

11. G. H. Cerda, La evaluación como experiencia total. Logros-objetivos-procesos, competencias y desempeños. (Bogotá: Cooperativa Editorial del Magisterio, 2000).

12. A. M. Pérez y Z. G. Bustamante, Evaluación escolar resultados o procesos. Investigación, reflexión y análisis.

13. A. Zambrano, Philippe Meirieu. Pedagogía, filosofía y política (Editorial Brujas: Argentina, 2011).

14. E. Morin, E. Ciurana y R. Motta, Educar en la era planetaria. Segunda edición (Barcelona: Editorial Gedisa, 2003). 
haciendo evaluaciones para ver cuántos se quedan y otros para ver cuántos se pasan, otros vemos a cuántos hay que ayudar"15.

El profesor advierte que las pruebas evaluativas buscan identificar sólo equivocaciones y errores en los estudiantes, ignorando sus potencialidades y sus fortalezas; desde la perspectiva de la evaluación formadora son importantes los procesos metacognitivos a través de los cuales el aprendiz hace visible su propio conocimiento, sus propias debilidades y fortalezas ${ }^{\mathbf{1 6}}$; es decir, es un proceso de autoevaluación permanente, que puede ser utilizado en beneficio del aprendizaje; fomentar el desarrollo de las potencialidades de un estudiante contribuye a alimentar su autoestima, su confianza y a evitar que mire la evaluación como aquella enemiga que sólo sirve para criticarlo y develar sus errores, conduciéndolo a desarrollar sentimientos de inferioridad e incapacidad.

Al respecto, algunos estudiantes advierten que las prácticas evaluativas de inglés reflejan sus fallas, mientras que otros sostienen que evidencian sus errores y las fortalezas, tal como los señalan los profesores; en ambos casos, es importante que conozcan sus aciertos y desaciertos para que ellos mismos hagan seguimiento de sus propios cambios y procesos ${ }^{17}$; sobre este tema un alumno sostiene lo siguiente:

\begin{abstract}
“Mmm ahorita que lo pienso creo que es más de los, los eh los defectos y en lo que falla el estudiante más que realmente lo que sabe. Como, como se dijo una evaluación lo puedo memorizar todo y listo, si? La memorizo y la hago y ya; pero si en una evaluación eh no se ve algo entonces ya está mostrando una debilidad en cierta, cierta, ciertas eh ciertas... en cierto tema entonces sí, si me eh sí creo que muestra más las debilidades que realmente las fortalezas"18.
\end{abstract}

El error que comete el alumno es valioso para monitorear lo que sucede en la clase y para aprovecharlo en beneficio no sólo de él mismo sino de sus compañeros; el reconocimiento que el profesor haga de las fortalezas del aprendiz puede tener efectos psicológicos que afecten positivamente su autoestima y su confianza y lo convenzan de que sí es posible lograr resultados satisfactorios, incluso en aquellas áreas o componentes del inglés en los cuales el alumno se considere débil.

Las pruebas deben generar reflexión permanente tanto en profesores como en estudiantes; al respecto un docente señala:

"[...] normalmente las evaluaciones no miden lo que es, no miden exactamente lo que yo quiero que midan, en un examen escrito por ejemplo es casi

15. Entrevista con profesores número 5, párrafo 25. Pasto, marzo de 2015.

16. M. Bordas y F. Cabrera. Estrategias de evaluación de los aprendizajes centrados en el proceso. Revista Española de Pedagogía. Año LIX, 218 (2001): 25-48, http: //ceeafime.wikispaces.com/file/view/ recurso16\%5B1\%5D.pdf (6 de febrero de 2014).

17. A. M. Pérez y Z. G. Bustamante, Evaluación escolar resultados o procesos. Investigación, reflexión y análisis.

18. Entrevista con estudiantes número 2, párrafo 33. Pasto, marzo de 2015. 
que imposible evaluar todo lo que los estudiantes aprendieron y todo lo que los estudiantes dejaron de aprender, en cambio por ejemplo si yo tengo una evaluación oral allí si yo puedo medir más exactamente lo que los estudiantes aprendieron y lo que no aprendieron, porque yo tengo la posibilidad de hacer el intercambio, la evaluación es injusta, entonces si por mí fuera yo no haría evaluaciones porque yo sé que las evaluaciones en lugar de contribuir a que el proceso de enseñanza aprendizaje se desarrolle, lo que hace en algunos casos es frenar, si el estudiante saca malas notas ya se predispone, ya no estudia, ya se desmotiva, ya se retira"19.

Se puede apreciar en estas revelaciones que el docente no confía totalmente en las prácticas evaluativas, pues éstas siempre son incompletas e injustas y de las cuales incluso se podría prescindir, en algunas ocasiones; desde luego que en el sistema actual de educación y evaluación, desistir de las evaluaciones sería para algunos como "dejar a la deriva" el aprendizaje del estudiante; casi no nos hemos detenido a pensar acerca de lo que pasaría con dicho aprendizaje, si no es evaluado: ¿los estudiantes ya no aprenderían? ¿Disminuiría su aprendizaje? ¿Disminuiría la calidad del sistema educativo?, estos y otros interrogantes están aún sin respuestas y esperan ser investigados.

Para algunos estudiantes, perder las pruebas de inglés y no tener las respuestas correctas son los factores que más les preocupa en las evaluaciones; es comprensible esta opinión si se considera que, infortunadamente, casi siempre la promoción a otro semestre o a otro nivel depende no necesariamente del aprendizaje logrado por el alumno, sino del promedio de calificaciones obtenido, una visión eminentemente cuantitativa y positivista; otro entrevistado va un poco más allá y señala que "le preocupa llegar a la práctica docente con deficiencias gramaticales, sintácticas, de vocabulario y de pronunciación del inglés"; de hecho, este estudiante está más preocupado por el saber y por su desempeño como futuro profesional que por la calificación de las pruebas mismas; aunque no parezca relevante, un entrevistado da gran importancia al tiempo para desarrollar las evaluaciones, por el temor a no responder las preguntas completamente; la idea de establecer un tiempo igual para todos es una herencia positivista que desconoce los parámetros culturales y contextuales y las particularidades del estudiante; al respecto, un profesor entrevistado afirma que él mismo realiza con anterioridad la prueba para calcular el tiempo requerido; sin embargo, hay que recordar que el profesor es quien, por lo general, elabora el examen y es quien tiene la respuesta o cree tenerla, por lo tanto, el tiempo que requiere el alumno y el docente para terminar una prueba no es el mismo.

Los profesores concuerdan en la existencia de ciertos factores que afectan al alumno en las prácticas evaluativas, tales como: las situaciones personales, pánico escénico, el nerviosismo, el ruido, la distracción, pruebas sorpresa, entre otras. Con respecto a las pruebas sin previo aviso, un docente indica:

19. Entrevista con profesores número 8, párrafo 21. Pasto, marzo de 2015. 
[...] "El estudiante eh... no se le haya avisado con tiempo de que había un proceso evaluativo y llegue de repente; es una manera para que el estudiante esté preparado, para que esté preparado ante circunstancias de la vida; no estamos preparados; yo les digo "muchachos, mañana, hoy, vamos a hacer una prueba; la evaluación tiene que ser continua porque el conocimiento es continuo" $[\ldots]^{20}$.

Este docente se declara en favor de la evaluación sorpresa ya que tiene aspectos positivos, como el hecho de mantener al alumno alerta ante cualquier eventualidad, tal como ocurre en la vida misma; sin embargo, esta clase de evaluación también es asociada con el ejercicio de poder como un mecanismo de control y de sometimiento que conlleva a un condicionamiento perverso en el peor de los casos: "si no atienden, hago examen" revela un profesor entrevistado, quien utiliza la evaluación como una amenaza y como una forma de ejercer violencia en el aula, en donde uno es el oprimido y otro es el opresor 21.

Los profesores sostienen que están satisfechos con los resultados obtenidos por los estudiantes en las pruebas de inglés; otros afirman no estarlo porque las evaluaciones no revelan realmente lo que saben; "hay quienes pasan y no merecían y hay quienes se quedan y merecían pasar, ante lo cual el docente no puede hacer nada" señala un docente; según esta opinión el profesor debe ser un sujeto neutral ante los resultados de las pruebas obtenidas por el alumno, es decir, debe respetar y ajustarse a lo que "le dice la nota", una posición eminentemente positivista que contrasta con la posición constructivista, en la que tanto el alumno como el profesor deben ser sujetos participantes y comprometidos, en este caso, con la evaluación y sus resultados para hacer los ajustes necesarios y lograr una mejora del proceso de enseñanza y aprendizaje; un docente señala que el buen estudiante merece estímulos; sin embargo, quien más los necesita es quien tiene dificultades de aprendizaje más visibles; no hacer nada es ser cómplice de ese fracaso. Otro docente afirma que la solución al problema del aprendizaje siempre recae en el alumno, nunca en la calidad del programa, del profesor, de la evaluación u otros aspectos; cuesta mucho creer que el único que no se equivoca en el aula es el profesor.

Por el contrario, los estudiantes no están completamente satisfechos con los resultados obtenidos en las pruebas de inglés, porque obtienen notas que no merecen, es decir, la calificación no compensa su esfuerzo; así lo evidencia una entrevistada al señalar:

"pues digamos que algunas materias que ha pasado como que no tan satisfecha porque...digamos uno mira el esfuerzo que uno ha hecho, porque hay materias que uno les pone más empeño por que digamos no es mi fuerte y tengo que superarlo, pues yo lo tomo de esa manera, entonces como que al final recibo una mala nota o una nota que no reflejo tanto esfuerzo, entonces digo; pero

20. Entrevista con profesores número 7, párrafo 23. Pasto, marzo de 2015.

21. Paulo Freire, Pedagogía del oprimido (México: Siglo XXI Editores S.A., 2005). 
qué me pasó, hay ciertas notas como lo que diría mejor dicho para que me colocaron esa nota, de qué me sirve esa nota o me colocaron un tres no más por pasarme y yo me esforcé, mucho, mucho, mucho y qué pasó con todo el esfuerzo que hice o sea uno diría; qué injusta esa nota o sea no valoraron, solamente se dedicaron al conocimiento y todo eso y no valoraron digamos el esfuerzo que uno hace a veces o el esfuerzo que a veces no hace o sea es si hay ciertas materias con las que no me he sentido cómoda en la evaluación al final"22.

La estudiante no encuentra relación entre el número obtenido en las calificaciones y su esfuerzo por aprender; la función social de la evaluación se apoya en concepciones cuantitativas y acumulativas del aprendizaje, la tradición asociacionista y los modelos conductistas o neoconductistas ${ }^{23}$; esto se advierte en la importancia que se ha dado al número y en la concepción de que la enseñanza es lineal y jerárquica, por lo cual se habla de nivel de aprendizaje y se hace énfasis en la medida del mismo. Otro participante más insatisfecho aún, sostiene que las pruebas de inglés, a pesar de ser necesarias en la universidad, lo desaniman y hacen que se sienta ignorante en un mundo donde equivocarse "no es permitido sino penalizado"; la opinión del estudiante conlleva a reflexionar acerca del carácter nocivo que pueden tener los exámenes, sobre todo cuando se observa el error del alumno como una manifestación de anormalidad, a tal punto de que él mismo crea que no es normal y que no está capacitado para tener éxito ni en la escuela ni en la vida misma; la idea de que hay dos clases de estudiantes (buenos y malos) merece ser reconsiderada; en su lugar, es preciso reconocer que cada alumno tiene dotes únicas ${ }^{24}$. Un estudiante afirma que el profesor enseña con palabras sencillas pero evalúa con palabras confusas; esta opinión reitera una vez más la utilización de la evaluación como instrumento de poder y como mecanismo para seleccionar y confundir al alumno. Por otra parte, las pruebas, infortunadamente, han sido concebidas como mecanismos de control, así, el profesor controla al alumno, el director al profesor, las entidades educativas al director y el sistema educativo a las entidades.

Los docentes sostienen que las pruebas están orientadas, por una parte, hacia la repetición, la gramática y la pronunciación; sobre este particular, un profesor aduce lo siguiente: "Para mí ambas cosas son importantes (memorización y gramática) porque hay casos que tienen que, tienen que memorizar porque en el inglés el vocabulario exacto o estructura es estructura exacta yo no puedo cambiarla en algunos casos"25. Esta opinión puede inscribirse en el campo del conocimiento declarativo que se enfoca en la exactitud, el dato, el principio, la explicación y la regla; son frecuentes las correcciones del profesor en torno a aspectos como el uso de las terceras personas del singular; el uso adecuado

22. Entrevista con profesores número 11, párrafo 27. Pasto, marzo de 2015.

23. C. Coll, Martín, E., T. Mauri, M. Miras, J. Onrubia, I. Solé, y A. Zabala, El constructivismo en el aula. (Barcelona: Editorial Graó, Des Serveis Pedagógics Francesc Tárrega, 2007).

24. P. Senge, La quinta disciplina: Escuelas que aprenden (Bogotá: Editorial Norma, 2002).

25. Entrevista con profesores número 8, párrafo 26. Pasto, marzo de 2015. 
de los verbos auxiliares para formular preguntas y para negar oraciones; la pronunciación de consonantes como p, t, k; los pasados de los verbos regulares, así como los elementos morfosintácticos de la lengua; desde luego que esta clase de errores pueden corregirse de manera inmediata, siempre y cuando no afecten significativamente la comunicación; recuérdese que en la vida cotidiana nadie habla un idioma perfectamente, incluso los hablantes nativos del inglés omiten los verbos auxiliares o las estructuras gramaticales en el uso informal de esa lengua.

Los dicentes, por su parte, atestiguan también que las pruebas se basan en la memoria, la repetición de información, lectura, fluidez y comprensión; el aprendizaje de un idioma extranjero, en este caso del inglés, tiene en cuenta el conocimiento declarativo, el conocimiento procedimental y pocas veces el actitudinal o de valores; tal vez por eso no es extraño que en las prácticas evaluativas se exija repetición y aplicación de la información; estos son legados y rezagos que en su momento dejaron los métodos de Gramática y Traducción (reglas), Audiolingual (hábitos y repetición), Método Directo (uso real del idioma) y el Enfoque Comunicativo, entre otros; es una herencia ecléctica cuya adecuada aplicación promete un aprovechamiento en favor del estudiante.

\section{OPERATIVIDAD DE LAS PRUEBAS}

En esta subcategoría se estudió la parte operativa de las evaluaciones, incluyendo los tipos de pruebas, su elaboración, frecuencia y procedimientos, entre otros aspectos. Los profesores, por su parte, revelan que las prácticas evaluativas se llevan a cabo de manera continua, tal como lo establece la función pedagógica de la evaluación; un docente advierte:

"No, yo no sé si me apego mucho a lo que dice el estatuto estudiantil de la universidad o sea que la evaluación debe ser continua, entonces no tengo fechas así definidas para el primer parcial, no. La idea es hacerla o sea después de terminar un tema y pues eh, sinceramente hay días y depende como te digo de los estudiantes. Si los estudiantes son muy molestosos y poco atienden o se nota como cierta indiferencia en la clase entonces ahí es donde más se aplica la evaluación"26.

Probablemente se tenga la impresión de que entre más se evalúe al estudiante más aprende, lo cual no necesariamente resulta cierto; si no se toman decisiones importantes después de cada evaluación quizás los resultados van a ser los mismos. La evaluación mide tanto al evaluador como al evaluado ${ }^{27}$, en este sentido, habría que suponer entonces que a mayor frecuencia de evaluaciones mejor sería el desempeño no sólo del alumno sino también del docente, puesto que cada prueba serviría como escenario de retroalimentación para ambos. Por otra parte, la tendencia de los profesores a elaborar sus propios exámenes, sugiere que las prácticas evaluativas

26. Entrevista con profesores número 1, párrafo 3. Pasto, marzo de 2015.

27. A. M. Pérez y Z. G. Bustamante, Evaluación escolar resultados o procesos. Investigación, reflexión y análisis. 
son contextualizadas y se ajustan a las realidades y necesidades del estudiante; no obstante, la elaboración de pruebas de inglés constituye un reto importante por cuanto implica tener conocimiento teórico sobre el tema; por ejemplo, es necesario conocer los principios básicos de la evaluación de una lengua: practicidad, confiabilidad, validez, autenticidad ${ }^{28}$; uno de los riesgos en la elaboración de pruebas evaluativas es confiar demasiado en la experiencia y olvidar ese componente teórico, "la teoría sin la acción es ingenua y la acción sin la teoría es ciega"29.

Los alumnos, al igual que los profesores, también expresan que las prácticas evaluativas en inglés son continuas y se ajustan a la reglamentación establecida por la universidad; esta tendencia resulta muy saludable por cuanto permite un acercamiento al proceso (siempre inacabado y cambiante) del aprendizaje del estudiante, pero no es suficiente si no se toman decisiones a partir de los resultados obtenidos; no sólo se evalúa algo (contenidos) sino para algo (mejora) ${ }^{30}$; en otras palabras, la evaluación debe ir más allá del cuestionario y las respuestas, para avanzar hacia las decisiones que favorezcan al aprendizaje.

Los profesores también manifiestan que evalúan predominantemente cada quince días y cuando estiman necesario; otro docente sostiene que evalúa a los estudiantes en la fechas programadas y al finalizar el semestre; se insinúa en esta declaración una preferencia por la evaluación sumativa que se hace en tiempos preestablecidos; un encuestado afirma que realiza una evaluación permanente mediante la observación y el monitoreo continuo en todas las actividades de clase, evidenciando así la aplicación de la función pedagógica de la evaluación, es decir orientada a la comprensión, al mejoramiento y a la toma de decisiones sobre la enseñanza y el aprendizaje.

Según la experiencia de los estudiantes, los docentes prefieren evaluarlos en las fechas de exámenes, al finalizar una unidad, al finalizar el semestre, cada quince días, cada mes, algunos lo hacen cada día y también cuando es necesario; se infiere que las evaluaciones son frecuentes, pero también se advierte que la evaluación ocurre como un elemento aislado del proceso de enseñanza y aprendizaje del inglés y al finalizar ciertos períodos de instrucción o ciclos, cuando los eventuales errores, tanto de enseñanza como de aprendizaje, ya han sido cometidos; es preciso indicar que no se trata de abundar en exámenes ni en calificaciones, sino de valorar (en el sentido cualitativo) y vigilar el proceso continuamente, en busca de irregularidades.

En relación con el tipo de evaluaciones que se aplican a los estudiantes para evaluar su aprendizaje del inglés, el examen escrito sobresale como práctica evaluativa, seguida de las pruebas orales, trabajos individuales, trabajos en grupo y los ensayos, según señalan los profesores; con menor frecuencia se utilizan

28. H. D. Brown, Teaching by Principles: An Interactive Approach to Language Pedagogy (New York: Pearson Education, 2007).

29. A. M. Pérez y Z. G. Bustamante, Evaluación escolar resultados o procesos. Investigación, reflexión y análisis.

30. C. Coll, Martín, E., T. Mauri, M. Miras, J. Onrubia, I. Solé, y A. Zabala, El constructivismo en el aula. 
las presentaciones guiadas y las presentaciones libres. Se puede observar el reconocimiento y la aceptación que han tenido y aún tienen el examen escrito y el examen oral en el campo de la evaluación del aprendizaje del inglés. Un profesor indica que utiliza otras prácticas evaluativas tales como: portafolios, conversatorios y talleres, jigsaw activities (actividades de rompecabezas) una vez por semestre y seminario alemán, una vez por semestre; se advierte en este profesor una tendencia a utilizar la evaluación alternativa y variada que favorece al estudiante y al proceso de aprendizaje.

Como puede apreciarse, existen creencias eclécticas entre profesores y estudiantes en relación con las características de las pruebas utilizadas en esta Unidad Académica. Las opiniones sobre el uso de los exámenes que ya vienen elaborados en los libros de texto están divididas; algunos profesores entrevistados consideran que sí los utilizan porque son elaborados por expertos y que pueden ser objeto de algunas adaptaciones; otros docentes creen que no se deben utilizar porque son muy limitados en muchos aspectos por cuanto estas clases de exámenes son elaborados para ser aplicados en cualquier lugar del mundo donde se aprenda el inglés como lengua extranjera, es decir, se basan en la generalización propia del pensamiento positivista; tales pruebas son elaboradas en otros contextos quizás para un "estudiante ideal" desconociendo las particularidades de las regiones y de los países; de ahí la importancia de reconocer el valor del conocimiento situado ${ }^{31}$ que nos ayuda a acercarnos mejor a la objetividad, a lo que no está preestablecido por otros, es decir, a lo no previsible; eso es, precisamente, lo que hacen las pruebas elaboradas por los mismos docentes, siempre y cuando sigan unos lineamientos teóricos básicos.

Los estudiantes afirman no estar de acuerdo con las pruebas de inglés que vienen con los libros de texto porque son homogéneas y no pueden adaptarse a todas las necesidades y contextos; indican que sólo le sirven al docente para cumplir con la obligación de evaluar, pero que debido a su proveniencia de otras latitudes, no siempre pueden revelar su aprendizaje.

\section{CARACTERIZACIÓN DE LAS PRUEBAS}

Esta subcategoría aborda aspectos atinentes a los temas evaluados en las pruebas, los criterios de evaluación y las modalidades, entre otros aspectos.

Los profesores manifiestan en su mayoría que utilizan las rúbricas como mecanismo para buscar equilibrio, justicia y equidad en las evaluaciones y obtener la mayor objetividad posible. Es necesario aclarar que las rúbricas no sólo se refieren a la inclusión del valor de cada pregunta en el examen; al menos deben contemplar los siguientes aspectos: a) los criterios o variables que se tienen en cuenta para valorar la actuación del alumno (fluidez, pronunciación, vocabulario); b) los niveles de cada criterio que pueden ser descriptivos (distinguido, eficiente, no eficiente) o numéricos (5-4-3-2-1);y c) la descripción de cada nivel que corresponde

31. D. J. Haraway, Ciencia, cyborgs y mujeres. La invención de la naturaleza (Madrid: Cátedra, 1995). 
a detallar los rasgos distintivos para determinar el grado de perfección ${ }^{32}$; a juzgar por las prácticas evaluativas analizadas en esta investigación, los profesores hacen uso parcial de las rúbricas, pues no se han implementado en su totalidad.

Al igual que los profesores, los estudiantes también reconocen que en las pruebas evaluativas por lo general sí se expresan al menos los valores de cada pregunta, aunque no siempre se describen en detalle las rúbricas o los objetivos de cada prueba.

En la gran mayoría de los exámenes de inglés analizados no se indican ni el objetivo de la prueba ni las rúbricas para su calificación, contrario a lo que expresan los profesores y algunos estudiantes; si bien es cierto, los profesores tienen autonomía para elaborar y calificar los exámenes, los estudiantes también tienen el derecho de conocer las condiciones bajo las cuales van a ser evaluados; las rúbricas son guías de puntaje que describen el grado en el que un aprendiz está ejecutando un proceso o producto y contribuyen a disminuir la subjetividad ${ }^{33}$; una prueba cuyas reglas de juego son desconocidas para los estudiantes se convierte en un arma de doble filo y crea escenarios de duda y confusión.

Aunque parezca contradictorio, a veces los docentes utilizan prácticas evaluativas cuyas preguntas poco tienen que ver con el contenido estudiado en las clases (tal como lo señaló un estudiante en una entrevista); en las pruebas analizadas en esta investigación, tales contenidos sí se ven reflejados en los exámenes; evaluar lo que no se ha estudiado en clase constituiría un acto de injusticia con el estudiante. En cuanto a la modalidad de pruebas se refiere, se observa mayoritariamente el uso de la práctica evaluativa individual, de hecho, los diez exámenes analizados se hicieron bajo esta modalidad, evidenciando así, la preferencia de los profesores por la evaluación individual, aislada, sin ayuda de apuntes, libros o la de los compañeros; en este sentido, el examen se convierte en una especie de rendición de cuentas, ante lo cual el alumno se ve obligado a veces a "aprender" al pie de la letra y en el peor de los casos a hacer trampa. En las prácticas evaluativas analizadas se nota el predominio de la evaluación del conocimiento de tipo declarativo (dato, norma, fecha, reglas) es decir, el saber qué ; también se evalúa el conocimiento procedimental (acciones, habilidades, procedimientos) o saber hacer; en realidad, estos dos tipos de conocimiento son inherentes al aprendizaje de una lengua extranjera; no obstante, se ignora casi por completo el valor del conocimiento actitudinal, el cual poco a poco ha ido ganando terreno en muchas áreas del conocimiento y en el campo de la evaluación, toda vez que tiene en cuenta el componente afectivo y humano del evaluado, incluyendo sus necesidades, sus sentimientos, sus actitudes y su esfuerzo, entre otros aspectos.

Es importante tener en cuenta que los principios de validez y confiabilidad son diferentes en el campo cuantitativo y en el campo cualitativo; la literatura

32. J.M. Nieto, Evaluación sin exámenes. Medios alternativos para comprobar el aprendizaje (Madrid: Editorial CCS, 2005).

33. F. Díaz-Barriga y R. G. Hernández, Estrategias docentes para un aprendizaje significativo. Una interpretación constructivista (Mexico: McGraw-Hill Interamericana, 2002). 
positivista tradicional (enfoque cuantitativo) se refiere a la validez como la facultad de medir lo que se tiene que medir; así, "la validez interna" de una investigación viene dada por la relación causa-efecto es decir, X causa Y pero no Z; "la validez externa" se orienta hacia la generalización de los hallazgos; desde la mirada cuantitativa la confiabilidad tiene que ver con la igualdad de resultados que se puede obtener en varios estudios independientemente del lugar y del tiempo de su realización; desde la mirada cualitativa (postpositivista), la validez se refiere al hecho de que los resultados "reflejen" una imagen lo más completa posible, clara y representativa (sentido pleno) de la realidad o situación estudiada; los estudios cualitativos poseen un alto nivel de validez derivado de su modo de recoger la información y de las técnicas de análisis que usan; la confiabilidad desde la mirada postpositivista está orientada hacia el nivel de concordancia interpretativa entre diferentes observadores, evaluadores o jueces del mismo fenómeno ${ }^{34}$. En las pruebas analizadas en esta búsqueda, se advierte una concepción cuantitativa de estos dos principios, por cuanto se exige del estudiante información precisa y detallada, de aspectos gramaticales y morfosintácticos.

Por otra parte, se advierte el predominio del tipo de test con base en "discrete points" que evalúa las habilidades lingüísticas de forma separada, como una muestra de la huella dejada por el método Audio-lingual.

\section{Conclusiones}

Los resultados de la investigación realizada evidencian la existencia de creencias eclécticas sobre las prácticas evaluativas en inglés, tanto en los profesores como en los estudiantes que participaron en el estudio. Algunos docentes advierten la presencia de la subjetividad y la preferencia por ciertos estudiantes en los exámenes, sin embargo, otros profesores admiten que la evaluación se hace en igualdad de condiciones de tiempo, lugar y contenidos; por su parte, los estudiantes reconocen que en las pruebas subyacen los prejuicios, la subjetividad, la desigualdad, la empatía y el estado de indefensión del alumno quien rara vez se queja o sienta su voz de protesta, quizás por temor a posibles retaliaciones; igualmente, aducen que el género, el buen comportamiento de los estudiantes y la trampa (copia) tienen incidencia en las calificaciones; además, señalan que solamente confían en las pruebas de falso o verdadero y no en las preguntas de respuesta abierta.

En relación con las dificultades de las pruebas, los profesores admiten que las pruebas de inglés son en ocasiones confusas, poco confiables y sostienen que no hay reflexión sobre los resultados de las mismas; advierten que se avalúa aprendizaje de memoria a corto plazo y que la inasistencia tiene efectos negativos en las pruebas; utilizan la evaluación sorpresa como mecanismo no solo de evaluación

34. M. Martínez, Validez y confiabilidad en la metodología cualitativa, Paradigma, 27(2) (2006): 07-33, en http://www.scielo.org.ve/scielo.php?script=sci_arttext\&pid=S1011-22512006000200002\&lng=es\& nrm=iso (7 de mayo de 2015). 
sino de control. Las pruebas evidencian, en su orden, énfasis en la repetición, la gramática, la pronunciación y la fluidez; no obstante, hay opiniones divididas, por cuanto algunos profesores manifiestan estar satisfechos con la evaluación que llevan a cabo, mientras que otros no lo están. Los estudiantes aducen que las dificultades más sensibles en las pruebas son el nerviosismo, el miedo al error, el miedo a perder las pruebas, la insatisfacción por los resultados y el tiempo para desarrollar los exámenes.

En cuanto a la operatividad de las pruebas, los profesores creen que éstas se hacen de manera continua y en fechas preestablecidas; predominan los exámenes escritos los cuales son frecuentemente tomados de los libros utilizados en las diferentes asignaturas; de igual manera, se cree que entre más evaluaciones se hagan mayor será el aprendizaje de los estudiantes. Por su parte los alumnos creen que las evaluaciones se hacen de manera continua y en fechas establecidas cuando el error ya se ha cometido; no consideran que abundar en exámenes implique mayor aprendizaje.

Con respecto a la caracterización de las pruebas de inglés, el análisis de las mismas muestra el predominio de la prueba individual y del conocimiento declarativo; en las pruebas no se indican las rúbricas con base en las cuales se va a evaluar a los estudiantes; finalmente, se advierte en los exámenes la influencia de los principios de validez y confiabilidad desde la perspectiva cuantitativa.

En resumen, las creencias de los profesores y de los estudiantes sobre los exámenes evidencian la influencia propia del positivismo; los participantes sostienen que el examen revela, casi con certeza, lo que el estudiante sabe o no sabe; en otras palabras, el resultado de la prueba es la representación de lo que ocurre en la mente del estudiante y pocas veces se tiene en cuenta sus situaciones personales, es decir, predomina el concepto de generalización propio del positivismo: todos reciben la misma enseñanza, luego todos deben aprender lo mismo. Finalmente, es preciso señalar que la subjetividad está presente en las pruebas evaluativas, pero no basta con reconocerlo, no hacer nada ante el fracaso del alumno en los exámenes es ser cómplice de ese fracaso; es preciso reflexionar sobre lo que sucede en los exámenes, por cuanto este insumo constituye una oportunidad para hacer retroalimentación tanto de la enseñanza como del aprendizaje, es decir, permite a profesores y a alumnos ser críticos y autocríticos ante las realidades y exigencias de una sociedad del conocimiento que exige otras visiones más inclusivas y más flexibles.

Investigaciones posteriores sobre el tema descrito en este artículo, podrían incluir análisis de la aplicación de pruebas con una caracterización más equilibrada de los componentes cualitativo y cuantitativo, con una operatividad que permita tener en cuentas las necesidades de los estudiantes con el fin de consolidar unas políticas que enfrenten y solucionen las dificultades propias de la evaluación. 


\section{REFERENCIAS BIBLIOGRÁFICAS}

Bachman, L.F., Fundamental Considerations in Language Testing. Oxford: Oxford. University Press, 1990.

Bordas M. y Cabrera, F., Estrategias de evaluación de los aprendizajes centrados en el proceso. Revista Española de Pedagogía. Año LIX, 218 (2001): 25-48, http: //ceeafime.wikispaces.com/file/view/ recurso16\%5B1\%5D.pdf. (6 de febrero de 2014).

Brown, H. D., Teaching by principles: An interactive Approach to Language Pedagogy. New York: Pearson Education, 2007.

Cerda, G. H., La evaluación como experiencia total. Logros-objetivos-procesos, competencias y desempeños. Bogotá: Cooperativa Editorial Magisterio, 2000.

Coll, C., Martín, E., Mauri, T., Miras, M., Onrubia, J., Solé, I., y Zabala, A., El constructivismo en el aula. Barcelona: Editorial Graó, Des Serveis Pedagógics Francesc Tárrega, 2007.

Deleuze, G. y Guattari, F., Rizoma. Medellín: Editorial Oveja Negra, 1976.

Díaz-Barriga, F. y Hernández, R. G., Estrategias docentes para un aprendizaje significativo. Una interpretación constructivista. México: McGraw-Hill Interamericana, 2002.

Entrevista con estudiantes número 1, párrafo 25. Pasto, marzo de 2015.

Entrevista con estudiantes número 2, párrafo 33. Pasto, marzo de 2015.

Entrevista con profesores número 1, párrafo 3. Pasto, marzo de 2015.

Entrevista con profesores número 11, párrafo 27. Pasto, marzo de 2015.

Entrevista con profesores número 2, párrafo 4. Pasto, marzo de 2015.

Entrevista con profesores número 5, párrafo 25. Pasto, marzo de 2015.

Entrevista con profesores número 7, párrafo 23. Pasto, marzo de 2015.

Entrevista con profesores número 8, párrafo 21. Pasto, marzo de 2015.

Entrevista con profesores número 8, párrafo 26. Pasto, marzo de 2015.

Freire, Paulo, Pedagogía del oprimido. México: Siglo XXI Editores S.A. 2005.

Haraway, D. J., Ciencia, cyborgs y mujeres. La invención de la naturaleza. Madrid: Cátedra, 1995.

Martínez, M., "Validez y confiabilidad en la metodología cualitativa”. Paradigma, 27(2) (2006): 07-33, en http://www.scielo.org.ve/scielo.php?script=sci_arttext\&pid=S1011-22512006000200002\&lng=es\&nrm =iso (7 de mayo de 2015).

Morin, E., Ciurana, E. y Motta, R., Educar en la era planetaria. Segunda edición. Barcelona: Editorial Gedisa, 2003.

Morin, E., Introducción al pensamiento complejo. Barcelona: Editorial Gedisa, 2011.

Morin, L., Méthodologie de L'histoire de vie. Québec: Institute supérieur des sciences humaines, Université Laval Coll, instruments de travail No. 13 (1974): 19. 
Nieto, J.M., Evaluación sin exámenes. Medios alternativos para comprobar el aprendizaje. Madrid: Editorial CCS, 2005.

Pérez, A. M. y Bustamante, Z. G., Evaluación escolar resultados o procesos. Investigación, reflexión y análisis crítico. Bogotá: Cooperativa Editorial del Magisterio, 2002.

Ricard, M., Bo, R. M. y, Climent, C.I., Propuesta de análisis fenomenológico de los datos obtenidos en la entrevista, UT. Revista de Ciències de l'Educació, Juny (2010): 113-133. http://pedagogia.fcep.urv.cat/revistaut (29 de abril de 2014).

Sánchez, A. T., “Aproximación a un estudio genealógico de la evaluación educativa en Colombia, segunda mitad del siglo XX”. Tesis doctoral. Centro de Estudios Avanzados en Niñez y Juventud Cinde - Universidad de Manizales, 2008.

Senge, Peter, La quinta disciplina: escuelas que aprenden. Bogotá: Editorial Norma, 2002.

Zambrano, A., Philippe Meirieu. Pedagogía, filosofía y política. Editorial Brujas: Argentina, 2011. 